\title{
Risk factors for Toxoplasma gondii infection among pregnant women from the State of Tocantins, Northern Brazil
}

\author{
Élvio Machado da Rocha ${ }^{[1]}$, Carlos Wilson Gomes Lopes ${ }^{[2],}$ \\ Rafael Antonio Nascimento Ramos ${ }^{[3]}$ and Leucio Câmara Alves ${ }^{[3]}$
}

[1]. Faculdade de Medicina, Instituto Tocantinense Presidente Antônio Carlos, Araguaína, Tocantins, Brasil. [2]. Departamento de Medicina Veterinária, Universidade Federal Rural do Rio de Janeiro, Seropédica, Rio de Janeiro, Brasil. [3]. Departamento de Medicina Veterinária, Universidade Federal Rural de Pernambuco, Recife, Pernambuco, Brasil.

\begin{abstract}
Introduction: The prevalence of infection by Toxoplasma gondii and associated risk factors in pregnant women in Tocantins, Northern Brazil were assessed. Methods: Serological analysis was performed in 338 serum samples and an oral questionnaire was utilized to identify potential risk factors. Results: Anti-IgG antibodies against $T$. gondii were detected in 71\% (240/338) of the pregnant women. Prior contact with cats and meat handling were found to be associated with T. gondii infection. Conclusions: A considerable percentage (29\%) of women living in the Tocantins area remain susceptible to infection by the T. gondii protozoon, representing a serious public health risk.
\end{abstract}

Keywords: Toxoplasma gondii. Pregnant women. Risk factors.

Toxoplasmosis is an important parasitic disease caused by the obligate intracellular protozoan Toxoplasma gondii, which is distributed worldwide ${ }^{(1)}$. This protozoon can potentially affect several species of animals including humans, but only those belonging to the Felidae family may act as definitive hosts $^{(2)}$. Infections in humans occur after the ingestion of raw or uncooked meat containing tissue cysts or by the ingestion of water, soil, or vegetables contaminated with oocysts released by infected cats $^{(3)}$. In most cases, the infected humans do not present clinical signs; however, when infection occurs in pregnant women several health problems can affect the fetuses. The transmission of $T$. gondii to the fetus might result in mental retardation, seizures, blindness, hydrocephalus, cerebral calcification, chorioretinitis, and ultimately death ${ }^{(4)}$. However, in some cases the health problems remain unapparent until the second or third decade of life ${ }^{(5)}$.

In pregnant women, the prevalence of infection might vary widely with levels ranging from less than $1 \%$ to $92 \%{ }^{(6)}(7)(8)$. Recently, a study conducted in Tanzania reported a seroprevalence of $30.9 \%$ in pregnant women ${ }^{(9)}$. In general, the lowest prevalence has been reported in North America, whereas in Latin America,

Corresponding author: Dr. Rafael Antonio Nascimento Ramos. Laboratório de Doenças Parasitárias dos Animais Domésticos/UFRPE. Av. Dom Manoel de Medeiros s/n, Dois Irmãos, 52171-900 Recife, Pernambuco, Brasil.

Phone: 5581 3320-6422

e-mail: rafaelanramos10@yahoo.com.br

Received 6 March 2015

Accepted 4 May 2015 and particularly in countries such as Brazil, Colombia, and Cuba the prevalence of infection might reach $70 \%^{(10)(11)}$. The wide prevalence range is due to the different risk factors, which might influence the likelihood of infection by $T$. gondii in a given population (e.g., ingestion of undercooked meat, unpasteurized milk, or educational level) ${ }^{(5)(12)}$.

Several studies have assessed the seroprevalence and risk factors of $T$. gondii in humans from Iran. In this country, different prevalences have been reported because of the marked geographical and cultural differences; in addition, distinct patterns of risk factors have been described ${ }^{(13)(14)}$. A similar situation is observed in Brazil, which is a large country with different social and environmental profiles. For example, it has been demonstrated that contact with the soil constitutes the most important risk factor associated with $T$. gondii infection in the Southern region of Brazil ${ }^{(15)}$. In contrast, the direct contact with cats was shown to be highly associated with $T$. gondii infection in humans residing in the northeast region ${ }^{(10)}$. Considering the importance of this zoonotic infection and the lack of data in some regions, the aim of this study was to assess the seroprevalence and the risk factors associated with $T$. gondii infection in pregnant women living in the State of Tocantins, Northern Brazil.

A cross-sectional study was conducted using serum samples of 338 pregnant women who were attended through the public healthcare system in the municipalities of Araguaína ( $7^{\circ} 11^{\prime} 31^{\prime \prime} \mathrm{S}$ and $\left.48^{\circ} 12^{\prime} 28^{\prime \prime} \mathrm{W}\right)$ and Colinas do Tocantins ( $8^{\circ} 3^{\prime} 23^{\prime \prime} \mathrm{S}$ and $48^{\circ} 28^{\prime} 38^{\prime \prime} \mathrm{W}$ ) in the State of Tocantins, Northern Brazil.

Blood samples were collected from the cephalic veins of the subjects using sterile tubes without anticoagulant and the 
sera obtained were stored at $-20^{\circ} \mathrm{C}$ until serological analysis. This was subsequently performed using a microparticle immunoenzymatic assay [(MEIA), AxSYM® System kit, Abbott Laboratories], following the manufacturer's instructions.

To identify the risk factors associated with infection by T. gondii, questionnaire data were assessed using Epi Info software (CDC Epi Info ${ }^{\mathrm{TM}}$ ) and analyzed by Pearson's chi square and Fisher's exact tests. The significance level was set at 5\% and the confidence interval (CI) at 95\%.

In this study, samples of 338 pregnant women were analyzed. Of these, $4.7 \%(16 / 338)$ were below 19 years of age, $63 \%$ (213/338) were between 20 and 29, and 23.4\% (79/338) were over 30 years old.

Anti-immunoglobulin G (IgG) antibodies against T. gondii were detected in $71 \%(240 / 338)$ of the pregnant women analyzed in this study, whereas anti-immunoglobulin M (IgM) antibodies were not observed. Among the patients with positive titer, $4.1 \%$ (10/240) were illiterate, 48.7\% (117/240) had spent less than five years in school, 38.7\% (93/240) had spent more than five years in the school, and $8.3 \%$ (20/240) were graduated from faculty. No difference was observed between the level of education and positive detection of T. gondii $(\mathrm{p}=0.598)$. Of the positive samples, $11.2 \%$ (27/240) and 88.7\% (213/240) were obtained from pregnant patients originating from rural and urban areas, respectively $(p=0.78)$. In addition, no differences were observed between patients from the two municipalities screened. Overall, results of the risk factor analysis are shown in Table 1.

This study demonstrates that contact with cats and the handling of meat are associated with infection by $T$. gondii in pregnant women from the State of Tocantins, Brazil. The seroprevalence $(71 \%)$ detected herein was within the range of positivity previously reported in pregnant Brazilian women (from $31 \%$ to $91.6 \%)^{(12)}$. It is known that seroprevalence might

TABLE 1 - Risk factors associated with infection by Toxoplasma gondii in pregnant women living in the State of Tocantins, Northern Brazil.

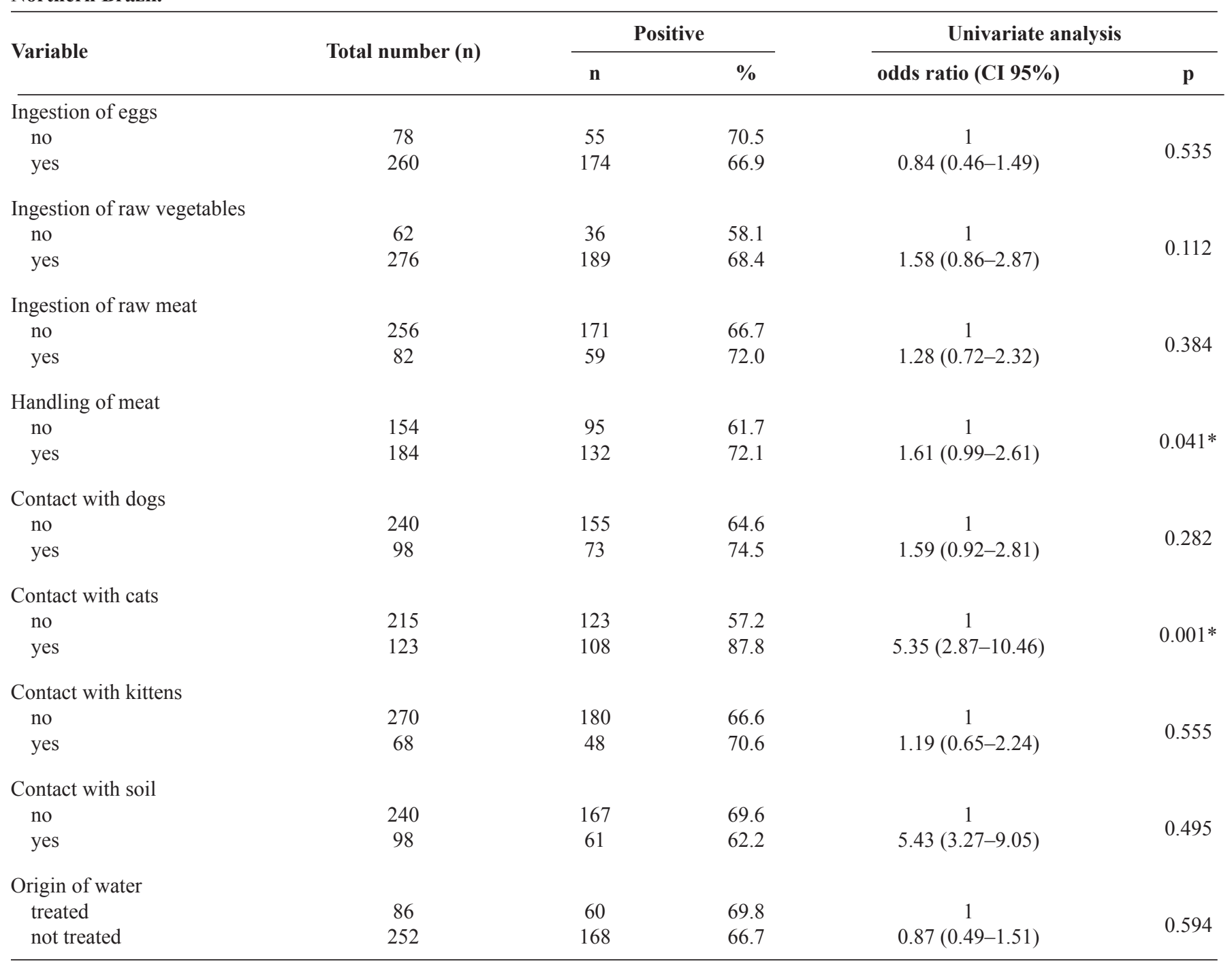

CI 95\%: confidence interval 95\%. *Risk factors associated with the infection by Toxoplasma gondii. 
vary widely in different parts of the world and may be influenced by various environmental and cultural factors ${ }^{(1)}$.

In contrast, this study did not find any correlation between T. gondii infection and age, level of educational, or origin of the pregnant women. Considering the lack of age association, the data obtained in this study suggests that in this area the infection probably occurred during childhood. The high infection rate during this period is likely due to the presence of stray cats and the play habits of children. In addition, although, it has previously been demonstrated that the educational level is an important risk factor for the occurrence of $T$. gondii infection, no association was detected in this study. In general, people with a higher educational level have more knowledge about this infection and the methods of its prevention ${ }^{(11)}$. As a similar lack of association was observed regarding the origin of samples (i.e., rural or urban areas), it is probable that the number of samples analyzed was small and lacked power to detect differences in infection rates across these parameters. However, in contrast to our results, the results of a previous study conducted in the municipality of Gurupi in the State of Tocantins indicated that a high prevalence of $T$. gondii infection in pregnant women was found to be related to age, as well as contact with raw meat, in natura milk intake, education level, working outside the home, and poor hygienic habits during meal preparation ${ }^{(12)}$.

In this study, certain evaluated feeding behaviors did not exhibit correlation with $T$. gondii infection. For example, a previous study suggested that the consumption of raw vegetables should be considered as a risk factor for infection ${ }^{(15)}$, but this was not relevant in our study. Additionally, toxoplasmosis has been associated with the ingestion of untreated water ${ }^{(14)}$, but this was also not observed here. It was suggested that toxoplasmosis might be transmitted to humans by the ingestion of sporulated oocysts in water and food ${ }^{(2)}$.

Instead, only the factors of contact with cats [odds ratio $(\mathrm{OR})=5.35, \mathrm{p}=0.01)]$ and meat handling $(\mathrm{OR}=1.61, \mathrm{p}=0.04)$ were associated with infection by $T$. gondii in the pregnant women living in the area assessed. Many studies demonstrated the importance of the presence of cats to the occurrence of infection by $T$. gondii. In fact, from an epidemiological point of view, the presence of cats acquires even more importance when these animals share the same environment with potential susceptible reservoirs, especially livestock animals and humans. In this context, the presence of a higher number of cats on a farm can increase the chances of infection of production animals, and in this case, the handling of (their) meat is considerably important in the epidemiology of this parasite. To date, in some Brazilian regions, domestic cats are fed with organs and parts of slaughtered animals; in addition, cats, albeit definitive T. gondii hosts, are maintained in the residences to control rats. Both behaviors might contribute to these animals becoming infected with $T$. gondii, and thus increasing the risk of infection in pregnant women.

In conclusion, in the population of pregnant women studied, $71 \%$ were anti-T. gondii IgG positive. These data indicate that a considerable percentage $(29 \%)$ of women living in this area remain susceptible to infection by this protozoon, which representing a serious risk for public health. Furthermore, our results demonstrate that contact with cats and the handling of meat are the main risk factors associated with infection by T. gondii in pregnant women from the State of Tocantins, Brazil.

\section{Ethical considerations}

All patients filled out a free and informed consent statement. In addition, an oral questionnaire was administered to collect information regarding lifestyle and pre-natal data. The present study was approved by the Ethics Committee of the Fundação de Medicina Tropical de Tocantins (protocol number 634127129/2009).

\section{CONFLICT OF INTEREST}

The authors declare that there is no conflict of interest.

\section{REFERENCES}

1. Dubey JP. History of the discovery of the life cycle of Toxoplasma gondii. Int J Parasitol 2009; 39:877-882.

2. Frenkel JK, Dubey JP. Toxoplasmosis and its prevention in cats and man. J Infect Dis 1972; 126:664-673.

3. Weiss LM, Kim K. Toxoplasma gondii: the model apicomplexan. Perspectives and methods. Academic Press: London; 2007.

4. Flatt A, Shetty N. Seroprevalence and risk factors for toxoplasmosis among antenatal women in London: a re-examination of risk in an ethnically diverse population. Eur J Public Health 2013; 23:648-652.

5. Jones JL, Lopez A, Wilson M, Schulkin J, Gibbs R. Congenital toxoplasmosis: a review. Obstet Gynecol 2001; 56:296-305.

6. Pappas G, Roussos N, Falagas ME. Toxoplasmosis snapshots: global status of Toxoplasma gondii seroprevalence and implications for pregnancy and congenital toxoplasmosis. Int J Parasitol 2009; 39:1385-1394.

7. Vaz RS, Thomaz-Soccol V, Sumikawa E, Guimarães ATB. Serological prevalence of Toxoplasma gondii antibodies in pregnant women from Southern Brazil. Parasitol Res 2010; 106:661-665.

8. Lopes AP, Dubey JP, Moutinho O, Gargaté MJ, Vilares A, Rodrigues M, et al. Seroepidemiology of Toxoplasma gondii infection in women from the North of Portugal in their childbearing years. Epidemiol Infect 2012; 140:872-877.

9. Mwambe B, Mshana SE, Kidenya BR, Massinde AN, Mazigo HD, Michael M, et al. Sero-prevalence and factors associated with Toxoplasma gondii infection among pregnant women attending antenatal care in Mwanza, Tanzania. Parasit Vectors 2013; 6:222.

10. Barbosa IR, Holanda CMCX, Andrade-Neto VF. Toxoplasmosis screening and risk factors amongst pregnant females in Natal, northeastern Brazil. Trans R Soc Trop Med Hyg 2009; 103:377-382.

11. Babaie J, Amiri S, Mostafavi E, Hassan N, Lotfi P, Esmaeili Rastaghi AR, et al. Seroprevalence and risk factors for Toxoplasma gondii infection among pregnant women in Northeast Iran. Clin Vaccine Immunol 2013; 20:1771-1773.

12. Silva MG, Câmara JT, Vinaud MC, Castro AM. Epidemiological factors associated with seropositivity for toxoplasmosis in pregnant women from Gurupi, State of Tocantins, Brazil. Rev Soc Bras Med Trop 2014; 47:469-475.

13. Youssefi MR, Sefidgar AA, Mostafazadeh A, Omran SM. Serologic evaluation of toxoplasmosis in matrimonial women in Babol, Iran. Pak J Biol Sci 2007; 10:1550-1552.

14. Saeedi M, Veghari GR, Marjani A. Seroepidemiologic evaluation of anti-Toxoplasma antibodies among women in north of Iran. Pak J Biol Sci 2007; 10:2359-2362.

15. Spalding SM, Amendoeira MRR, Klein CH, Ribeiro LC. Serological screening and toxoplasmosis exposure factors among pregnant women in South of Brazil. Rev Soc Bras Med Trop 2005; 38:173-177. 